\title{
Phenotypic heterogeneity in mycobacterial stringent response
}

\author{
Sayantari Ghosh¹, Kamakshi Sureka², Bhaswar Ghosh, Indrani Bose ${ }^{1 *}$, Joyoti Basu², Manikuntala Kundu²
}

\begin{abstract}
Background: A common survival strategy of microorganisms subjected to stress involves the generation of phenotypic heterogeneity in the isogenic microbial population enabling a subset of the population to survive under stress. In a recent study, a mycobacterial population of M. smegmatis was shown to develop phenotypic heterogeneity under nutrient depletion. The observed heterogeneity is in the form of a bimodal distribution of the expression levels of the Green Fluorescent Protein (GFP) as reporter with the gfp fused to the promoter of the rel gene. The stringent response pathway is initiated in the subpopulation with high rel activity.

Results: In the present study, we characterise quantitatively the single cell promoter activity of the three key genes, namely, mprA, sigE and rel, in the stringent response pathway with gfp as the reporter. The origin of bimodality in the GFP distribution lies in two stable expression states, i.e., bistability. We develop a theoretical model to study the dynamics of the stringent response pathway. The model incorporates a recently proposed mechanism of bistability based on positive feedback and cell growth retardation due to protein synthesis. Based on flow cytometry data, we establish that the distribution of GFP levels in the mycobacterial population at any point of time is a linear superposition of two invariant distributions, one Gaussian and the other lognormal, with only the coefficients in the linear combination depending on time. This allows us to use a binning algorithm and determine the time variation of the mean protein level, the fraction of cells in a subpopulation and also the coefficient of variation, a measure of gene expression noise.
\end{abstract}

Conclusions: The results of the theoretical model along with a comprehensive analysis of the flow cytometry data provide definitive evidence for the coexistence of two subpopulations with overlapping protein distributions.

\section{Background}

Microorganisms are subjected to a number of stresses during their lifetime. Examples of such stresses are: depletion of nutrients, environmental fluctuations, lack of oxygen, application of antibiotic drugs etc. Microorganisms take recourse to a number of strategies for survival under stress and adapting to changed circumstances [1-4]. A prominent feature of such strategies is the generation of phenotypic heterogeneity in an isogenic microbial population. The heterogeneity is advantageous as it gives rise to variant subpopulations which are better suited to persist under stress. Bistability refers to the appearance of two subpopulations with distinct phenotypic characteristics [5,6]. In one of the subpopulations, the expression of appropriate stress response genes is

\footnotetext{
* Correspondence: indrani@bosemain.boseinst.ac.in

'Department of Physics, Bose Institute, Kolkata, India

Full list of author information is available at the end of the article
}

initiated resulting in adaptation. There are broadly two mechanisms for the generation of phenotypic heterogeneity $[7,8]$. In "responsive switching" cells switch phenotypes in response to perturbations associated with stress. In the case of "spontaneous stochastic switching", transitions occur randomly between the phenotypes even in the absence of stress. Responsive switching may also have a stochastic component as fluctuations in the level of a key regulatory molecule can activate the switch once a threshold level is crossed $[1,3,5,6]$.

The pre-existing phenotypic heterogeneity, an example of the well-known "bet-hedging-strategy", keeps the population in readiness to deal with future calamities. Using a microfluidic device, Balaban et al. [9] have demonstrated the existence of two distinct subpopulations, normal and persister, in a growing colony of E. coli cells. The persister subpopulation constitutes a small fraction of the total cell population and is
C Biomed Central

C 2011 Ghosh et al; licensee BioMed Central Ltd. This is an Open Access article distributed under the terms of the Creative Commons Attribution License (http://creativecommons.org/licenses/by/2.0), which permits unrestricted use, distribution, and reproduction in any medium, provided the original work is properly cited. 
distinguished from the normal subpopulation by a reduced growth rate. Since killing by antibiotic drugs like ampicillin depends on the active growth of cell walls, the persister cells manage to survive when the total population is subjected to antibiotic treatment. The normal cells, with an enhanced growth rate are, however, unable to escape death. Once the antibiotic treatment is over, some surviving cells switch from the persister to the normal state so that normal population growth is resumed $[9,10]$. A simple theoretical model involving transitions between the normal and persister phenotypes explains the major experimental observations well $[9,11]$. In the case of environmental perturbations, Thattai and Oudenaarden [12] have shown through mathematical modeling that a dynamically heterogeneous bacterial population can under certain circumstances achieve a higher net growth rate conferring a fitness advantage than a homogeneous one. Mathematical modelling further shows that responsive switching is favoured over spontaneous switching in the case of rapid environmental fluctuations whereas the reverse is true when environmental perturbations are infrequent [7]. Another theoretical prediction that cells may tune the switching rates between phenotypes to the frequency of environmental changes has been verified in an experiment by Acar et al. [13] involving an engineered strain of S. cerevisiae which can switch randomly between two phenotypes. The major feature of all such studies is the coexistence of two distinct subpopulations in an isogenic population and their interconversions in the presence/absence of stress. Bistability, i.e., the partitioning of a cell population into two distinct subpopulations has been experimentally observed in a number of cases $[1,3,5]$. Some prominent examples include: lysis/lysogeny in bacteriophage $\lambda$ [14], the activation of the lactose utilization pathway in E. coli [15] and the galactose utilization genetic circuit in S. cerevisiae [16], competence development in B. subtilis $[6,17,18]$ and the stringent response in mycobacteria [19].

The mycobacterial pathogen M. tuberculosis, the causative agent of tuberculosis, has remarkable resilience against various physiological and environmental stresses including that induced by drugs [20-22]. On tubercular infection, granulomas form in the host tissues enclosing the infected cells. Mycobacteria encounter a changed physical environment in the confined space of granulomas with a paucity of life-sustaining constituents like nutrients, oxygen and iron [23,24]. The pathogens adapt to the stressed conditions and can survive over years in the so-called latent state. In vitro too, M. tuberculosis has been found to persist for years in the latent state characterised by the absence of active replication and metabolism [25]. Researchers have developed models simulating the possible environmental conditions in the granulomas. One such model is the adaptation to nutrient-depleted stationary phase [26]. The processes leading to the slowdown of replicative and metabolic activity constitute the stringent response. In mycobacteria, the expression of rel initiates the stringent response which leads to persistence. The importance of Rel arises from the fact that it synthesizes the stringent response regulator ppGpp (guanosine tetraphosphate) [27] and is essential for the long-term survival of $M$. tuberculosis under starvation [28] and for prolonged life of the bacilli in mice [29].

Key elements of the stringent response and the ability to survive over long periods of time under stress are shared between the mycobacterial species $M$. tuberculosis and M. smegmatis [30]. Recent experiments provide knowledge of the stress signaling pathway in mycobacteria linking polyphosphate (poly $\mathrm{P}$ ), the two-component system MprAB, the alternate sigma factor SigE and Rel [31]. In an earlier study [19], we investigated the dynamics of rel-gfp expression ( $g f p$ fused with rel promoter) in M. smegmatis grown upto the stationary phase with nutrient depletion serving as the source of stress. In a flow cytometry experiment, we obtained evidence of a bimodal distribution in GFP levels and suggested that positive feedback in the stringent response pathway and gene expression noise are responsible for the creation of phenotypic heterogeneity in the mycobacterial population in terms of the expression of rel$g f p$. Positive feedback gives rise to bistability $[5,6]$, i.e., two stable expression states corresponding to low and high GFP levels. We further demonstrated hysteresis, a feature of bistability, in rel-gfp expression. The mathematical model developed by us to study the dynamics of the stringent response pathway predicted bistability in a narrow parameter regime which, however, lacks experimental support. In general, to obtain bistability a gene circuit must have positive feedback and cooperativity in the regulation of gene expression. Recently, Tan et al. [32] have proposed a new mechanism by which bistability arises from a noncooperative positive feedback circuit and circuit-induced growth retardation. The novel type of bistability was demonstrated in a synthetic gene circuit. The circuit, embedded in a host cell, consists of a single positive feedback loop in which the protein product $\mathrm{X}$ of a gene promotes its own synthesis in a noncooperative fashion. The protein decay rate has two components, the degradation rate and the dilution rate due to cell growth. In the circuit considered, production of $X$ slows down cell growth so that at higher concentrations of $\mathrm{X}$, the rate of dilution of $\mathrm{X}$ is reduced. This generates a second positive feedback loop since increased synthesis of $\mathrm{X}$ proteins results in faster accumulation of the proteins so that the protein concentration is higher. The combination of two positive feedback 
loops gives rise to bistability in the absence of cooperativity. A related study by Klumpp et al [33] has also suggested that cell growth inhibition by a protein results in positive feedback.

\section{Results}

In this paper, we develop a theoretical model incorporating the effect of growth retardation due to protein synthesis [32,34]. We provide some preliminary experimental evidence in support of the possibility. In our earlier study [19], bimodality in the rel-gfp expression levels was observed. As a control, GFP expression driven by the constitutive $h s p 60$ promoter was monitored as a function of time. A single bright population was observed at different times of growth (Figure S4 of [19]). The unimodal rather than bimodal distribution ruled out the possibility that clumping of mycobacterial cells and cell-to-cell variation of plasmid copy number were responsible for the observed bimodal fluorescence intensity distribution of $\mathrm{rel}$ promoter driven GFP expression. In the present study, we perform flow cytometry experiments to monitor $m p r A-g f p$ and $s i g E-g f p$ expression levels. The distribution of GFP levels in each case is found to be bimodal. We determine the probability distributions of the two subpopulations associated with low and high expression levels at different time points in the three cases of mprA-gfp, sigE-gfp and rel-gfp expression. In each case, the total distribution is a linear combination of two invariant distributions with the coefficients in the linear combination depending on time. The results of hysteresis experiments are also reported.

\section{Mathematical modeling of the stress response pathway}

Figure 1 shows a sketch of the important components of the stress response pathway in M. smegmatis subjected to nutrient depletion $[19,31]$. The operon mprAB consists of two genes mprA and $m p r B$ which encode the histidine kinase sensor MprB and its partner the cytoplasmic response regulator MprA respectively. The protein pair responds to environmental stimuli by initiating adaptive transcriptional programs. Polyphosphate kinase 1 (PPK1) catalyses the synthesis of polyphosphate (poly $\mathrm{P})$ which is a linear polymer composed of several

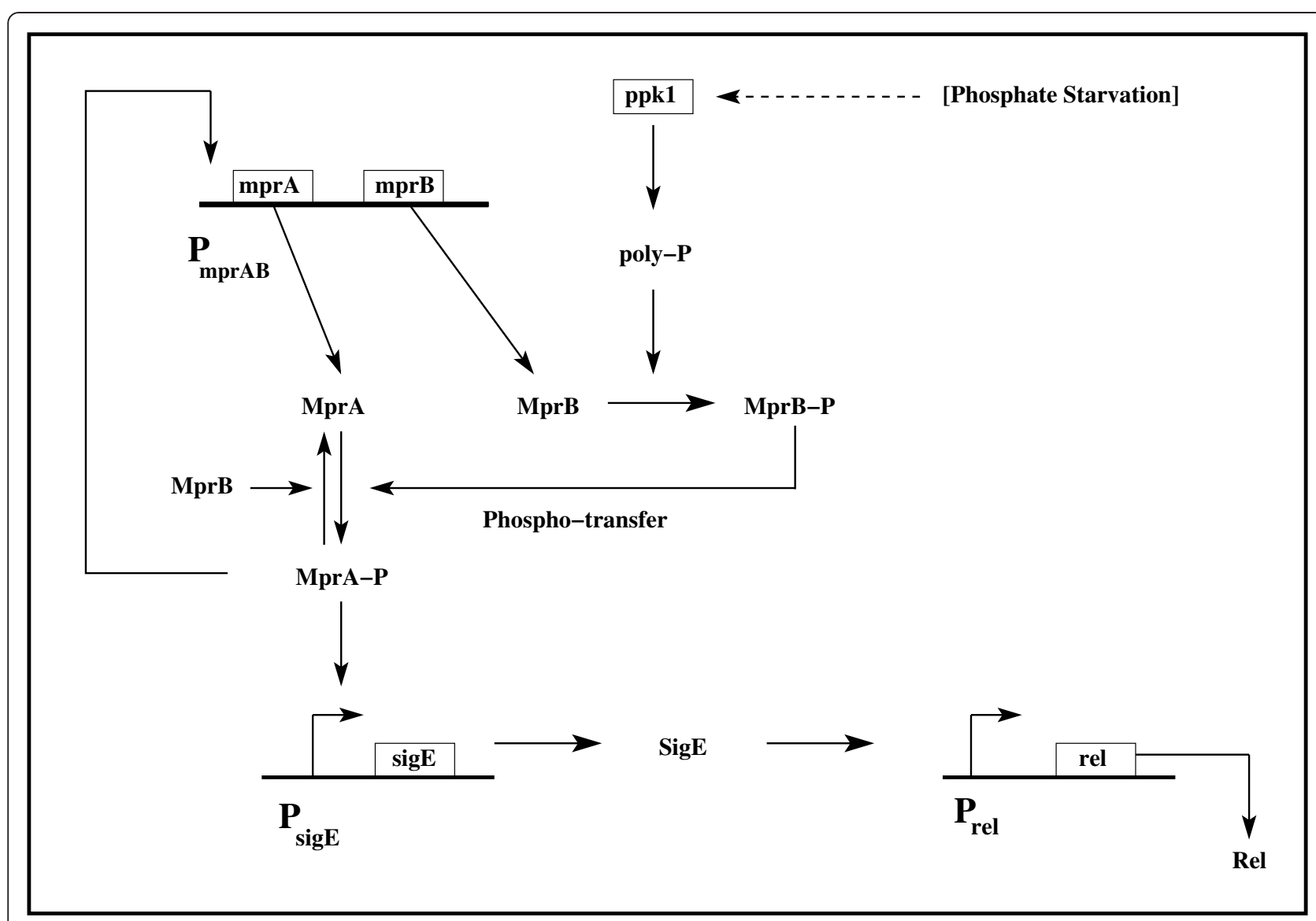

Figure 1 Schematic diagram of the stringent response pathway in M. smegmatis activated under nutrient depletion. MprB-P and MprA$\mathrm{P}$ are the phosphorylated forms of MprB and MprA respectively. Poly P serves as the phosphate donor in the conversion of MprB to MprB-P. 
orthophosphate residues. Mycobacteria possibly encounter a phosphate-limited environment in macrophages. Sureka et al. [31] proposed that poly P could play a critical role under ATP depletion by providing phosphate for utilisation by MprAB. A recent experiment [34] on a population of $M$. tuberculosis has established that the MTB gene $p p k 1$ is significantly upregulated due to phosphate starvation resulting in the synthesis of inorganic poliphosphate (poly P).

The two-component regulatory system SenX3-RegX3 is known to be activated on phosphate starvation in both M. smegmatis [35] and M. tuberculosis [34]. In the latter case, RegX3 has been shown to regulate the expression of $p p k 1$, a feature expected to be shared by $M$. smegmatis. In both the mycobacterial populations, poly $\mathrm{P}$ regulates the stringent response via the mprAsigE-rel pathway [31]. In our experiments, nutrient depletion possibly gives rise to phosphate starvation. On activation of the mprAB operon, MprB autophosphorylates itself with poly $\mathrm{P}$ serving as the phosphate donor $[31,34]$. The phosphorylated MprB-P phosphorylates MprA via phosphotransfer reactions. There is also evidence that MprB functions as a MprA-P (phosphorylated MprA) phosphatase. MprA-P binds the promoter of the $m p r A B$ operon to initiate transcription. A positive feedback loop is functional in the signaling network as the production of MprA brings about further MprA synthesis. The mprAB operon has a basal level of gene expression independent of the operation of the positive feedback loop. Once the mprAB operon is activated, MprA-P regulates the transcription of the alternate sigma factor gene $\operatorname{sig} E$, which in turn controls the transcription of rel. We construct a mathematical model to study the dynamics of the above signaling pathway. The new feature included in the model takes into account the possibility that the production of stress-induced proteins like MprA and MprB slows down cell growth. This effectively generates a positive feedback loop as explained in Refs. [32,34]. Figure 2(a) shows the mean amount of GFP fluorescence in the total mycobacterial population as measured in a flow cytometry experiment ( $m p r A$ promoter fused with $g f p$ ) versus time. Figure 2(a) shows the specific growth rate of the cell population versus time. The inset shows the experimental growth curve for the mycobacterial population. The growth was monitored by recording the absorbance values at $600 \mathrm{~nm}$ spectrophotometrically (see Methods). The specific growth rate at time $t$ is given by $\frac{1}{N(t)} \frac{d N(t)}{d t}$ where $N(t)$ is the number of mycobacterial cells at time $t$. Nutrient depletion limits growth and proliferation and culminates in the activation of stress response genes. It appears that in many cases rapid growth and stress response are mutually exclusive so that the production of a stress response protein gives rise to a slower growth rate [36]. The balance between the expressions of growth-related and stress-induced genes determines the cellular phenotype with respect to growth rate and stress response. Persister cells in both E. coli $[9,10]$ and mycobacteria $[21,22]$ have slow growth rates. In the case of $M$. smegmatis, we have already established that the slower growing persister subpopulation has a higher

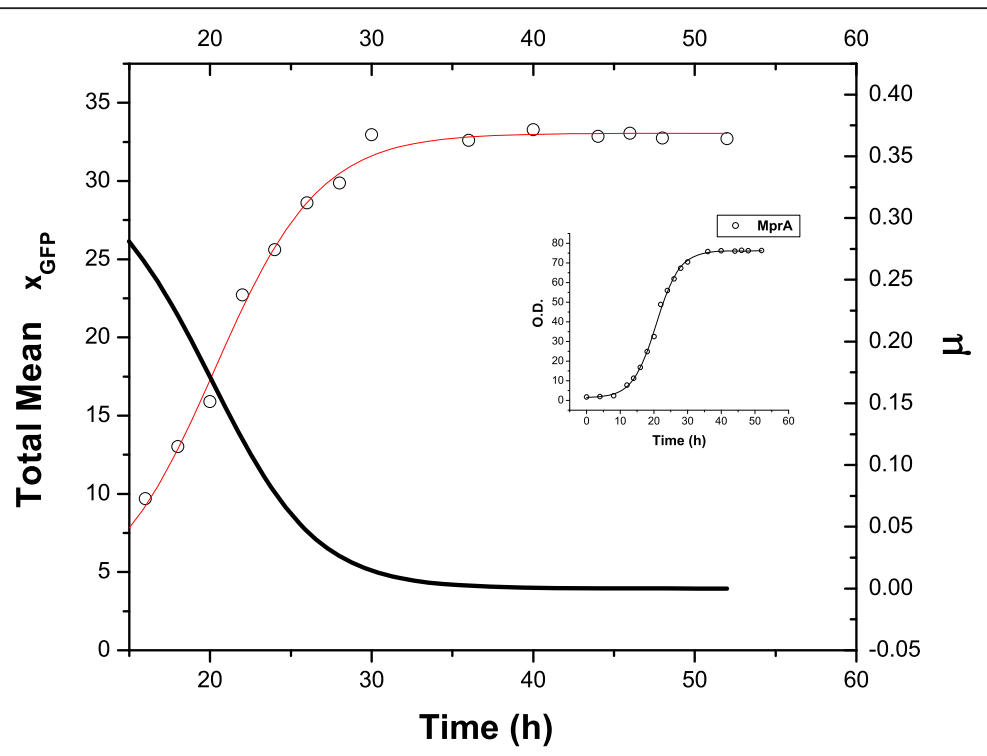

Figure 2 Growth retardation due to protein synthesis. (a) Mean amount of GFP fluorescence in the case of mprA promoter fused with gfp and (b) specific growth rate $\mu$ of mycobacterial population versus time in hours (h). The growth curve (optical density versus time) of the mycobacterial population is shown in the inset. 
level of Rel, the initiator of stringent response, as compared to the normal subpopulation [19]. The new addition to our mathematical model [19] involves nonlinear protein decay rates arising from cell growth retardation due to protein synthesis. We briefly discuss the possible origins of the nonlinearity and its mathematical form $[32,34]$. The temporal rate of change of protein concentration is a balance between two terms: rate of synthesis and rate of decay. The decay rate constant $\left(\gamma_{\text {eff }}\right)$ has two components: the dilution rate due to cell growth $(\mu)$ and the natural decay rate constant $(\gamma)$, i.e., $\gamma_{\text {eff }}=\mu+\gamma$ where $\mu$ is the specific growth rate. In many cases, the expression of a protein results in cell growth retardation $[32,34]$. The general form of the specific growth rate in such cases is given by

$$
\mu=\frac{\phi}{1+\theta x}
$$

where $x$ denotes the protein concentration and $\varphi, \theta$ are appropriate parameters. In Ref. [32], the expression for $\mu$ (Eq. (1)) is arrived at in the following manner. The Monod model [37] takes into account the effect of resource or nutrient limitation on the growth of bacterial cell population. The rate of change in the number of bacterial cells is

$$
\frac{d N}{d t}=\mu N
$$

where the specific growth rate $\mu$ is given by

$$
\mu=\mu_{\max } \frac{s}{k+s}
$$

In (3), $s$ is the nutrient concentration and $k$ the half saturation constant for the specific nutrient. When $s=$ $k$, the specific growth rate attains its half maximal value ( $\mu_{\max }$ is the maximum value of specific growth rate). The metabolic burden of protein synthesis affecting the growth rate is modeled by reducing the nutrient amount $s$ by $\in$, i.e.,

$$
\mu=\frac{\mu_{\max }}{1+\frac{k}{s(1-\epsilon)}}
$$

The magnitude of is assumed to be small and proportional to the protein concentration $x$. Following the procedure outlined in the Supplementary Information of [32], namely, applying Taylor's expansion to (4) and putting $\in=\lambda x$ ( $\lambda$ is a constant), one obtains the expression in Eq. (1) with $\phi=\frac{\mu_{\max } s}{s+k}$ and $\theta=\frac{k \lambda}{s+k}$.

Thus, the decay rate of proteins has the form $-\gamma_{\text {eff }} x=$ $-(\gamma+\mu) x$ where $\mu$ is given by Eq. (1). There are alternative explanations for the origin of the nonlinear decay term, e.g., the synthesis of a protein may retard cell growth if it is toxic to the cell [33]. In the case of mycobacteria, there is some experimental evidence of cell growth retardation brought about by protein synthesis. The response regulator MprA has an essential role in the stringent response pathway leading to persistence of mycobacteria under nutrient deprivation. Inactivation of the regulator in an mprA insertion mutant resulted in reduced persistence in a murine model but the growth of the mutant was proved to be significantly higher than that observed in the cases of the wild-type species $[38,39]$. Our experimental data (Figure 3) provide further support to the hypothesis that MprA synthesis leads to reduced specific growth rate. The data points represent GFP fluorescence intensity with $g f p$ fused to the mprA promoter. The GFP acts as a reporter of the mprA promoter activity culminating in MprA (also MprB) synthesis. The data points shown in Figure 3 are those that correspond to the growth period of 16-23 hours in Figure 2.

The data points are fitted by an expression similar to that in Eq. (1) with $\mu_{\max }^{G F P}=0.94$ and $\theta^{G F P}=0.317$. The differential equations describing the temporal rates of change of key protein concentrations in our model are described in the Additional File 1. Solving the equations, one finds the existence of bistability, i.e., two stable expression states in an extended parameter regime. Figures S1 A-C (Additional File 1) show the plots for bistability and hysteresis for the proteins MprA, SigE and Rel versus the autophosphorylation rate. In the deterministic scenario and in the bistable regime, all the

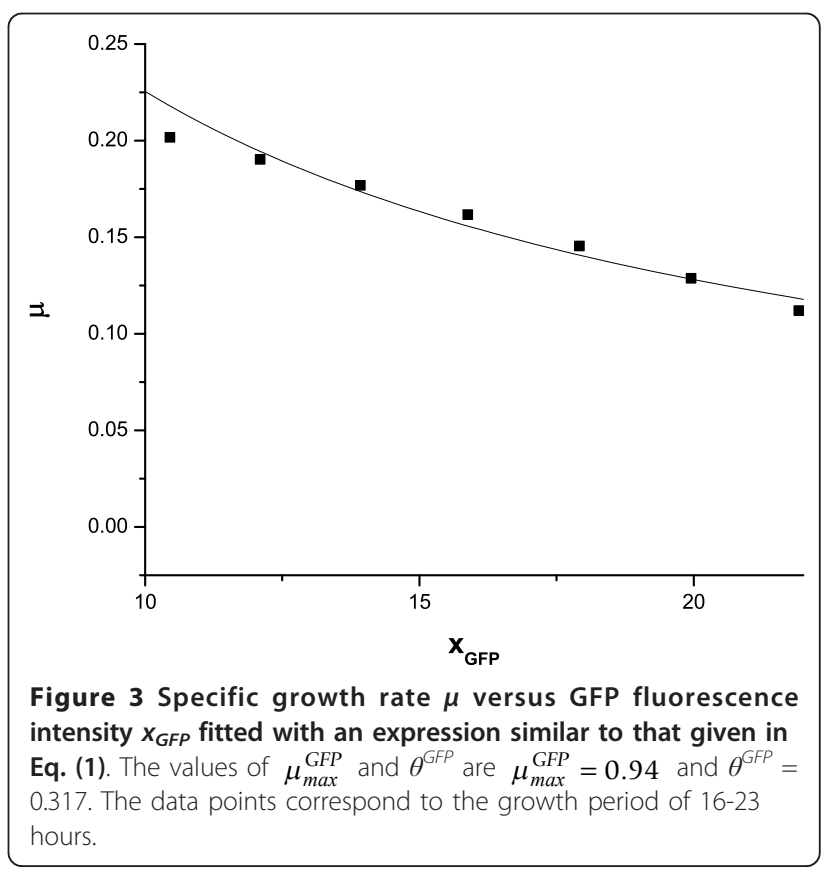


cells in a population are in the same steady state if exposed to the same environment and with the same initial state. The experimentally observed heterogeneity in a genetically identical cell population is a consequence of stochastic gene expression. The biochemical events involved in gene expression are inherently probabilistic $[40,41]$ in nature. The uncertainty introduces fluctuations (noise) around mean expression levels so that the single protein level of the deterministic case broadens into a distribution of levels. In the case of bistable gene expression, the distribution of protein levels in a population of cells is bimodal with two distinct peaks.

Bimodal Expression of $\mathrm{mprA}$, sigE and rel in M. smegmatis In the earlier study [19], we investigated the dynamics of rel transcription in individual cells of $M$. smegmatis grown in nutrient medium up to the stationary phase, with nutrient depletion serving as the source of stress. We employed flow cytometry to monitor the dynamics of green fluorescent protein (GFP) expression in $M$. smegmatis harboring the rel promoter fused to $g f p$ as a function of time. The experimental signature of bistaility lies in the coexistence of two subpopulations. We now extend the study to investigate the dynamics of $m p r A$ and sigE transcription in individual M. smegmatis cells in separate flow cytometry experiments. Figure 4(a) and 4(b) show the time course of mprAGFP and sigE-GFP expressions respectively as monitored by flow cytometry. In both the cases, the distribution of GFP-expressing cells is bimodal indicating the existence of two distinct subpopulations. In each case, the cells initially belong to the subpopulation with low GFP expression. The fraction of cells with high GFP expression increases as a function of time. The two subpopulations with low and high GFP expression are designated as $\mathrm{L}$ and $\mathrm{H}$ subpopulations respectively. In the stationary phase, the majority of the cells belong to the $\mathrm{H}$ subpopulation. The presence of two distinct subpopulations confirms the theoretical prediction of bistability.

We analysed the experimental data shown in Figure 4 and found that at any time point the distribution $P(x, t)$ of GFP levels in a population of cells is a sum of two overlapping and time-independent distributions, one Gaussian $\left(P_{1}(x)\right)$ and the other lognormal $\left(P_{2}(x)\right)$, i.e.,

$$
P(x, t)=C_{1}(t) P_{1}(x)+C_{2}(t) P_{2}(x)
$$

The coefficients $C_{i}^{\prime}$ 's $(\mathrm{i}=1,2)$ depend on time whereas $P_{1}(x)$ and $P_{2}(x)$ are time-independent. The general forms of $P_{1}(x)$ and $P_{2}(x)$ are,

$$
\begin{aligned}
& P_{1}(x)=\frac{\exp \left(-\left(\frac{x-x_{01}}{w_{01}}\right)^{2}\right)}{w_{01} \sqrt{\frac{\pi}{2}}} \\
& P_{2}(x)=\frac{\exp \left(-\frac{1}{2}\left(\frac{\ln x-x_{02}}{w_{02}}\right)^{2}\right)}{x w_{02} \sqrt{2 \pi}}
\end{aligned}
$$

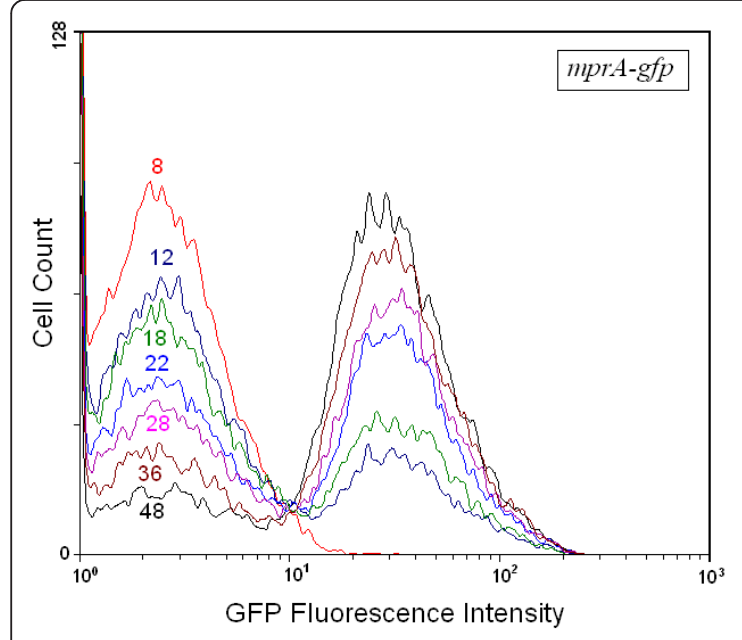

(a)

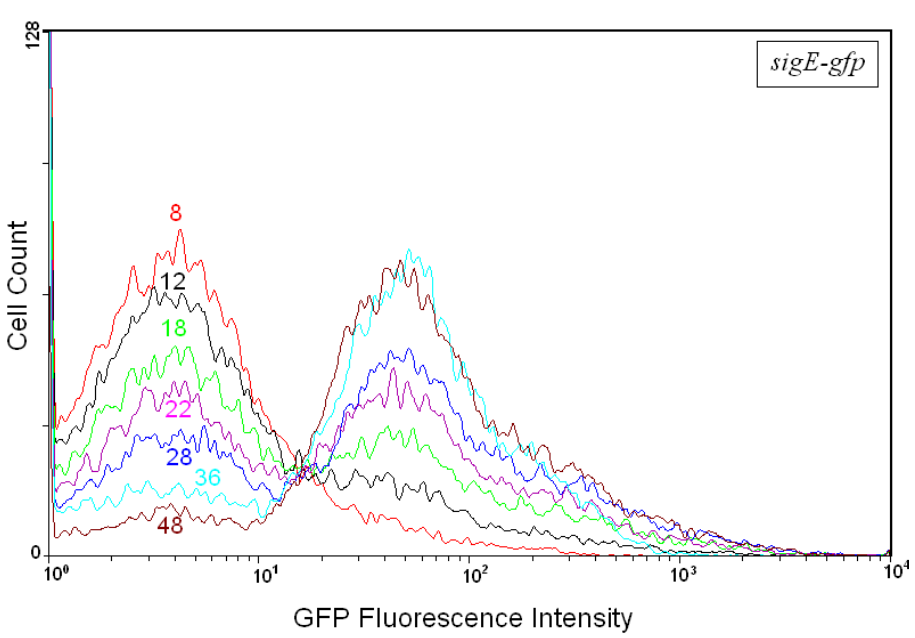

(b)

Figure 4 Time course of (a) mprA-gfp and (b) sigE-gfp expression. M. smegmatis harboring the appropriate promoter construct was grown for different periods of time (indicated in hours (h)) and the specific promoter-driven expression of GFP was monitored by flow cytometry. With time, there is a gradual transition from the $\mathrm{L}$ to the $\mathrm{H}$ subpopulation. 
Figures S2(a) and (b) in Additional File 1 illustrate the typical forms of the Gaussian and lognormal distributions. The Gaussian distribution has a symmetric form whereas the lognormal distribution is asymmetric and long-tailed. Figure 5 shows the experimental data for cell count versus GFP fluorescence intensity at selected time points in the cases when $g f p$ is fused with mprA and sigE promoters in separate experiments. The dotted curves represent the individual terms in the r.h.s. of Eq. (5) and the solid curve denotes the linear combination $P(x, t)$. The different parameters of $P_{1}(x)$ and $P_{2}(x)$ have the values $x_{01}=97.3366(145.86181) ; w_{01}=$ 103.0731 (154.67381); $x_{02}=5.95526(6.1171) ; w_{02}=$ 0.17618 (0.2509) when $g f p$ is fused with mprA (sigE). The ratio of the coefficients, $C_{1}(t) / C_{2}(t)$, has the value listed by the side of each figure. Figure S3 displays a similar analysis of the experimental data when $g f p$ is fused to the rel promoter.

In the earlier study [19], the total cell population was divided into $\mathrm{L}$ and $\mathrm{H}$ subpopulations depending on whether the measured GFP fluorescence intensity was less or greater than a threshold intensity. In the present study, we have obtained approximate analytic expressions for the distributions of GFP fluorescence intensity in the $\mathrm{L}$ and $\mathrm{H}$ subpopulations. The two distributions, Gaussian and lognormal, have overlaps in a range of fluorescence intensity values (Figure 5 and Figure S3 in Additional File 1). We next used the binning algorithm developed by Chang et al. [42] to partition the cells of the total population into two overlapping distributions, one Gaussian (Eq. (6)) and the other lognormal (Eq. (7)). At time $\mathrm{t}$, let $\mathrm{N}(\mathrm{t})$ be the total number of cells. For each cell, the data $x_{j}$ for the fluorescence intensity is used to calculate the ratios,

$$
g_{1}\left(x_{j}\right)=\frac{P_{1}\left(x_{j}\right)}{P_{1}\left(x_{j}\right)+P_{2}\left(x_{j}\right)}, g_{2}\left(x_{j}\right)=\frac{P_{2}\left(x_{j}\right)}{P_{1}\left(x_{j}\right)+P_{2}\left(x_{j}\right)}
$$

where $P_{1}(x)$ and $P_{2}(x)$ are the distributions in Eqs. (6) and (7). A random number $\mathrm{r}$ is generated and the cell $j$ is assigned to the $\mathrm{L}$ subpopulation if $0 \leq r<g_{1}\left(x_{j}\right)$, the cell belongs to the $\mathrm{H}$ subpopulation otherwise. Once the total population is partitioned into the $\mathrm{L}$ and $\mathrm{H}$ subpopulations, one can calculate the following quantities:

$$
\begin{aligned}
\omega_{i}(t) & =\frac{N_{i}(t)}{N(t)}(i=1,2) \\
\mu_{i}(t) & =\sum x_{j i}(t) / N_{i}(t) \\
\sigma_{i}^{2}(t) & =\sum\left(x_{j i}(t)-\mu_{i}(t)\right)^{2} /\left(N_{i}(t)-1\right)
\end{aligned}
$$

The indices $i=1,2$ correspond to the $\mathrm{L}$ and $\mathrm{H}$ subpopulations, $\omega_{i}(t)$ is the fraction of cells in the $i$ th subpopulation at time $\mathrm{t}, \mu_{i}(t)$ is the mean fluorescence intensity for the $i$ th subpopulation and $\sigma_{i}^{2}(t)$ the associated variance. Figure 6 shows the results of the data analysis. Figure 6(a) shows the plots of mean GFP fluorescence level for the $L$ subpopulation (basal level) versus time in the three cases of $g f p$ fused with the promoters of $m p r A$, sigE and rel respectively. Figure 6(b) displays the data for the fractions of cells, $\omega_{2}(t)$, versus time in the three cases and Figure 6(c) shows the transition rate versus time along with the coefficients of variation $\mathrm{CV}$ $(\mathrm{CV}=$ standard deviation/mean $)$ of the protein levels in the $\mathrm{L}$ subpopulation versus time.

Figure S4 (Additional File 1) shows the plots of mean GFP fluorescence level for the total population versus time in the three cases of $g f p$ fused with the promoters of $m p r A$, sigE and rel respectively. As in the case of the basal level versus time data (Figure 6(a)), the plots are sigmoidal in nature. We solved the differential equations of the theoretical model described in Additional File 1 and obtained the concentrations of MprA, MprB, SigE, MprA-P, MprB-P and GFP versus time. Some of these plots are shown in Figure S5 (Additional File 1) and reproduce the sigmoidal nature of the experimental plots. We note that the sigmoidal nature of the curves is obtained only when the non-linear nature of the degradation rate is taken into account.

As we have already discussed, the distribution of GFP levels in the mycobacterial cell population is a linear combination of two invariant distributions, one Gaussian and the other lognormal, with only the coefficients in the linear combination dependent on time. Friedman et al. [43] have developed an analytical framework of stochastic gene expression and shown that the steady state distribution of protein levels is given by the gamma distribution. The theory was later extended to include the cases of transcriptional autoregulation as well as noise propagation in a simple genetic network. While experimental support for gamma distribution has been obtained earlier [44], a recent exhaustive study [45] of the E. coli proteome and transcriptome with single-molecule sensitivity in single cells has established that the distributions of almost all the protein levels out of the 1018 proteins investigated, are well fitted by the gamma distribution in the steady state. The gamma distribution was found to give a better fit than the lognormal distribution for proteins with low expression levels and almost similar fits for proteins with high expression levels. We analysed our GFP expression data to compare the fits using lognormal and gamma distributions. For all the three sets of data ( $g f p$ fused with the promoters of $m p r A$, sigE and rel), the lognormal and gamma distribution give similar fits at the different time points. Figure S6 (Additional File 1) shows a comparison of 

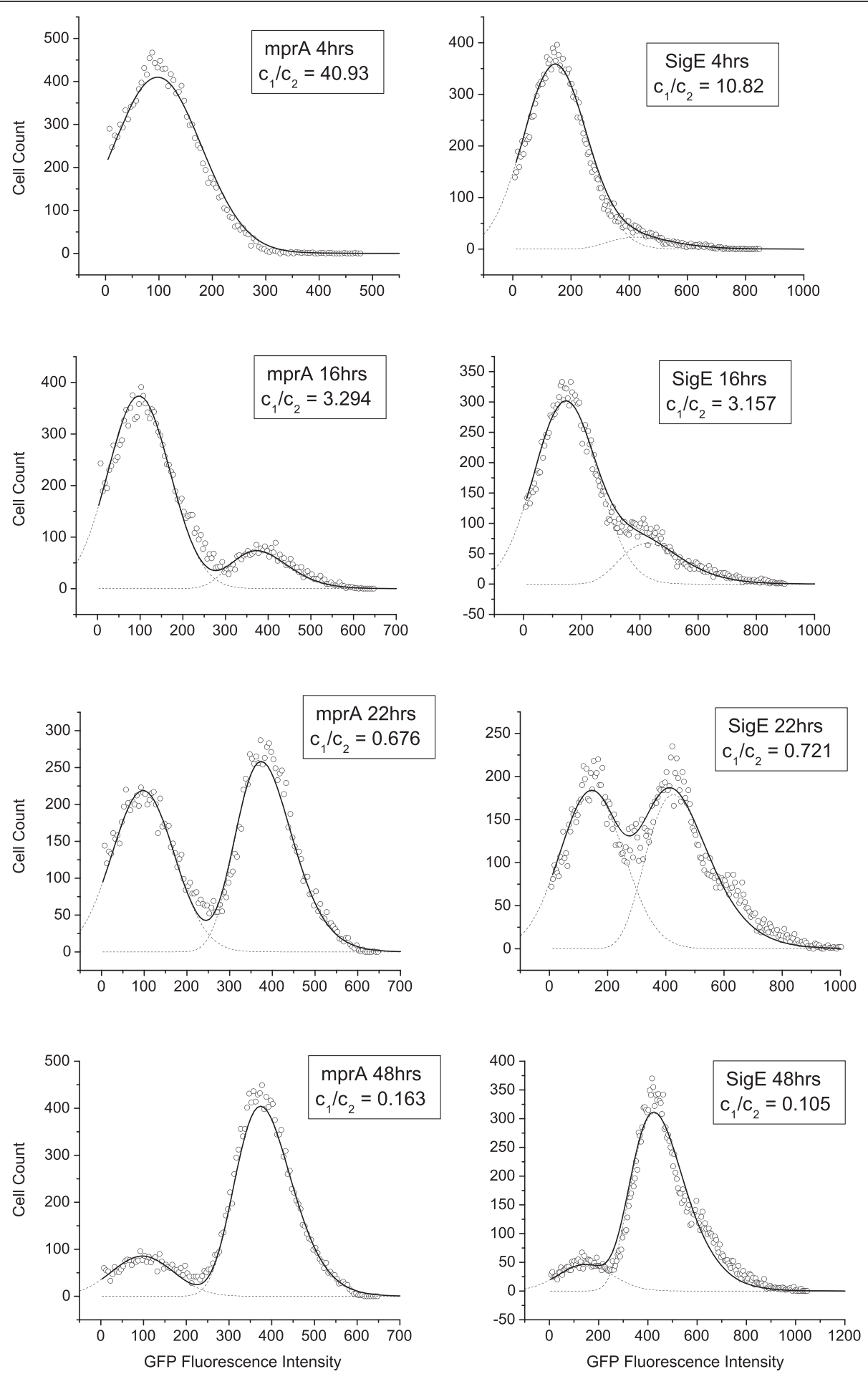

Figure 5 Fitting of data with two distributions. Experimental data for cell count versus GFP fluorescence intensity at selected time points when $g f p$ is fused with mprA and sigE promoters respectively. The solid curve represents $P(x, t)$ in equation (5) and the dotted curves are the individual terms on the r.h.s. 


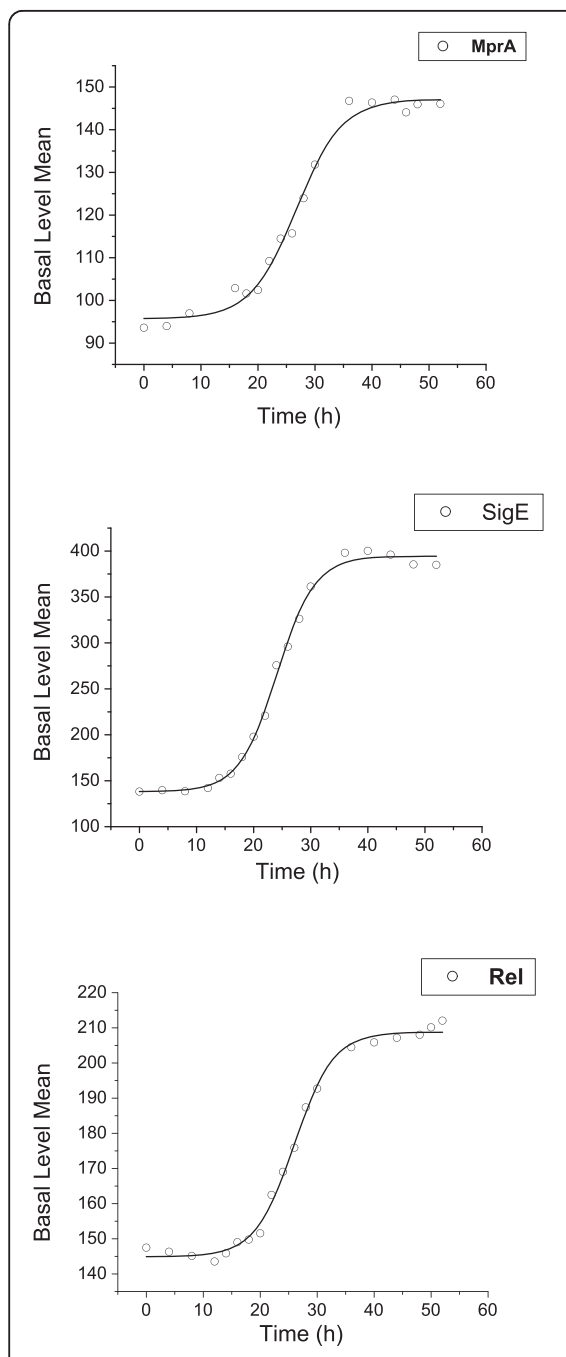

(a)
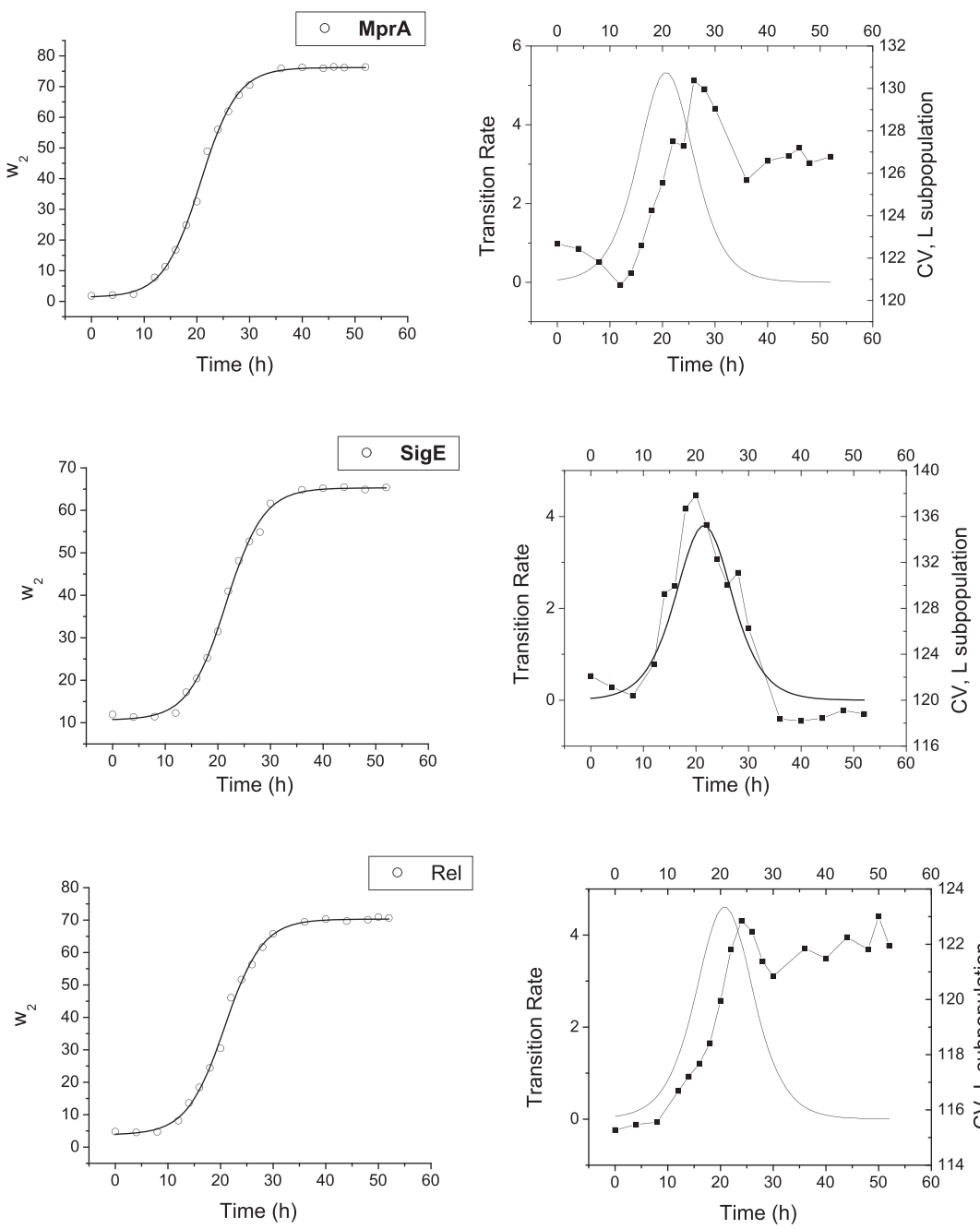

(b)

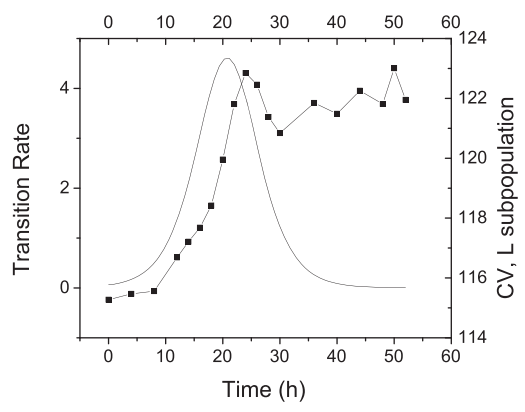

(c)

Figure 6 Analysis of the time course of gfp expression. (a) Mean protein level in $L$ subpopulation (basal level) versus time in hours in the three cases of gfp fused with $m p r A$, sigE and rel promoters respectively. (b) Fraction of cells $\omega_{2}(\mathrm{t})$ in the $\mathrm{H}$ subpopulation versus time in hours in the three cases. (c) Transition rate from the $\mathrm{L}$ to the $\mathrm{H}$ subpopulation and the $\mathrm{CV}$ (experimental data shown) of the protein levels in the $\mathrm{L}$ subpopulation versus time in hours in the three cases. The experimental data are analyzed using the binning algorithm to obtain the plots (a), (b) and (c).

the fits for the case of gfp-mprA. The lognormal appears to give a somewhat better fit than the gamma distribution, specially at the tail ends.

\section{Hysteresis in gfp expression}

Some bistable systems exhibit hysteresis, i.e., the response of the system is history-dependent. In the earlier study, experimental evidence of hysteresis was obtained with $g f p$ fused to the promoter of rel. The experimental procedure followed for the observation of hysteresis is as follows. In PPK-KO, the ppk1 knockout mutant, the $p p k 1$ gene was introduced under the control of the tet promoter. We grew PPK-KO carrying the tetracycline-inducible $p p k 1$ and rel-gfp plasmid in medium with increasing concentration of tetracycline (inducer). For each inducer concentration, the distribution of cells expressing $g f p$ was analysed by flow cytometry in the stationary phase (steady state) and the mean GFP level was measured. A similar set of experiments was carried out for decreasing concentrations of tetracycline. In the present study, hysteresis experiments in the manner described above were carried out in the two cases of $g f p$ fused to $m p r A$ and $s i g E$ promoters respectively. Figure 7 shows the hysteresis data (mean GFP fluorescence versus inducer concentration) in the two cases for increasing (branch going up) and decreasing (branch 
going down) inducer concentrations. The existence of two distinct branches is a confirmation of hysteresis in agreement with theoretical predictions (Figures S1 A-C). Figure 8 shows the GFP distributions in the stationary phase for two sets of experiments with different histories, one in which the inducer concentration is increased from low to a specific value (indicated as "Low" in black) and the other in which the same inducer concentration is reached by decreasing the inducer concentration from a high value (indicated as "High" in red). The distributions show that two regions of monostability are separated by a region of bistability. In the cases of monostability, the distributions with different histories more or less coincide. In the region of bistability, the distributions are distinct indicating a persistent memory of initial conditions.

\section{Discussion}

The development of persistence in microbial populations subjected to stress has been investigated extensively in microorganisms like E. coli and mycobacteria $[9,10,21,22,28,29]$. In an earlier study [19], we demonstrated the roles of positive feedback and gene expression noise in generating phenotypic heterogeneity in a population of M. smegmatis subjected to nutrient depletion. The heterogeneity was in terms of two distinct subpopulations designated as $\mathrm{L}$ and $\mathrm{H}$ subpopulations. The subpopulations corresponded to persister and nonpersister cell populations with the stringent response being initiated in the former. In the present study, we have undertaken a comprehensive single cell analysis of the expression activity of the three key molecular players in the stringent response pathway, namely, MprA, SigE and Rel. This has been done by fusing $g f p$ to the respective genes in separate experiments and monitoring the
GFP levels in a population of cells via flow cytometry. The distribution has been found to be bimodal in each case.

In our earlier study [19], with only the positive autoregulation of the mprAB operon taken into account, bistability was obtained in a parameter regime with restricted experimental relevance. The inclusion of the effective positive feedback loop due to growth retardation by protein synthesis gives rise to a considerably more extended region of bistability in parameter space. The persister cells with high stringent response regulator levels are known to have slow growth rates $[21,22,28,29]$. This is consistent with the view that stress response diverts resources from growth to stress-related functions resulting in the slow growth of stress-resistant cells [36]. Figure 2 and 3 provide experimental evidence that the mean intensity of GFP fluorescence monitoring $m p r A-g f p$ expression increases with time while the specific growth rate $\mu$ of the $M$. smegmatis population decreases in the same time interval. The reciprocal relationship between the two quantities is represented by an expression similar to that in Eq. (1). Since our knowledge of the detailed genetic circuitry involved in the stringent response is limited, we have not attempted to develop a model to explain the origin of cell growth retardation due to protein synthesis. Further experiments (e.g., sorting of the mycobacterial cell population into two subpopulations) are needed to provide conclusive evidence that increased protein synthesis retards cell growth. The stringent response pathway involving MprA and MprB is initiated when the mycobacterial population is subjected to stresses like nutrient depletion. There is now experimental evidence of complex transcriptional, translational, and posttranslational regulation of SigE

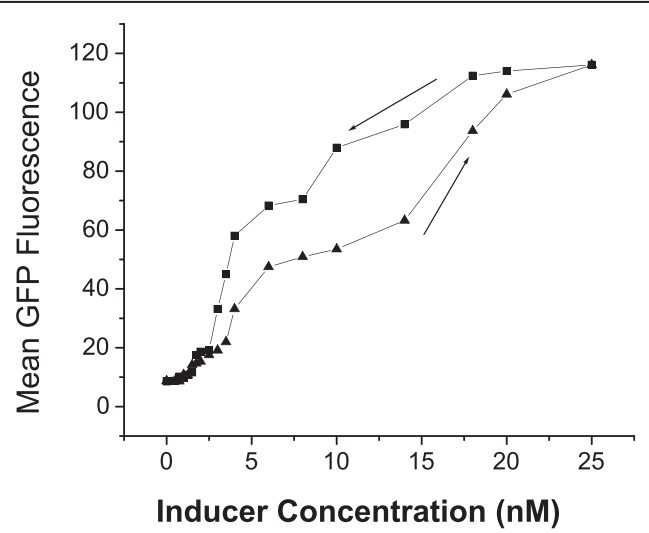

(a)

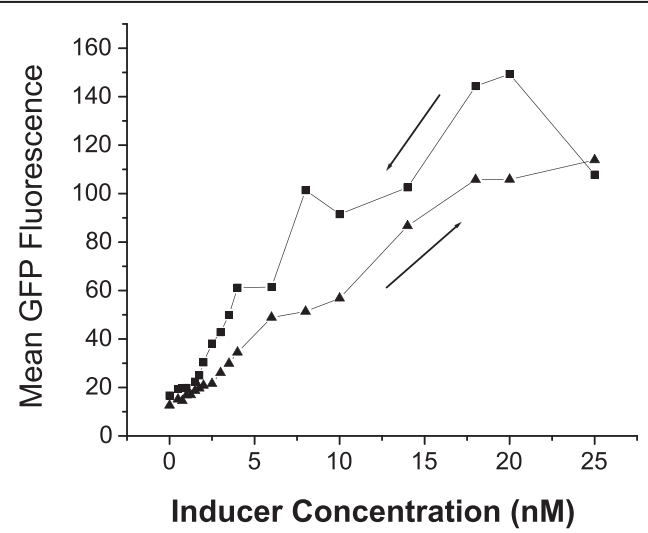

(b)

Figure 7 Hysteresis in gfp expression. The gene gfp is fused with (a) $m p r A$ and (b) sigE promoter. Filled triangles and squares represent the experimental data of mean GFP fluorescence with increasing and decreasing concentrations of tetracycline inducer respectively. 

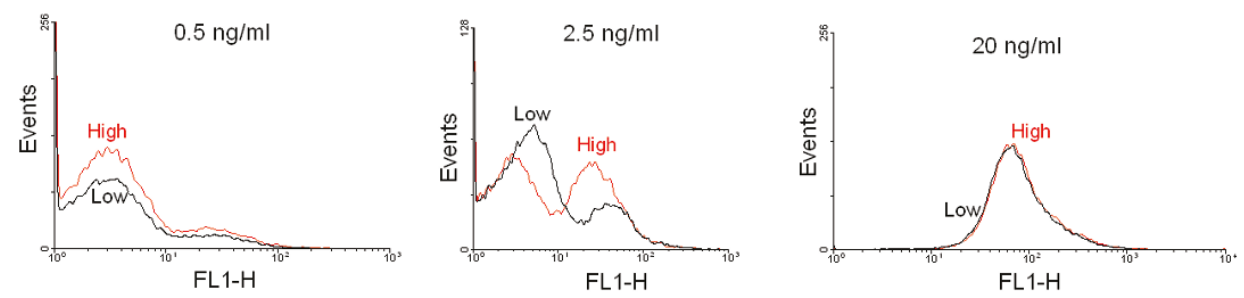

(a)
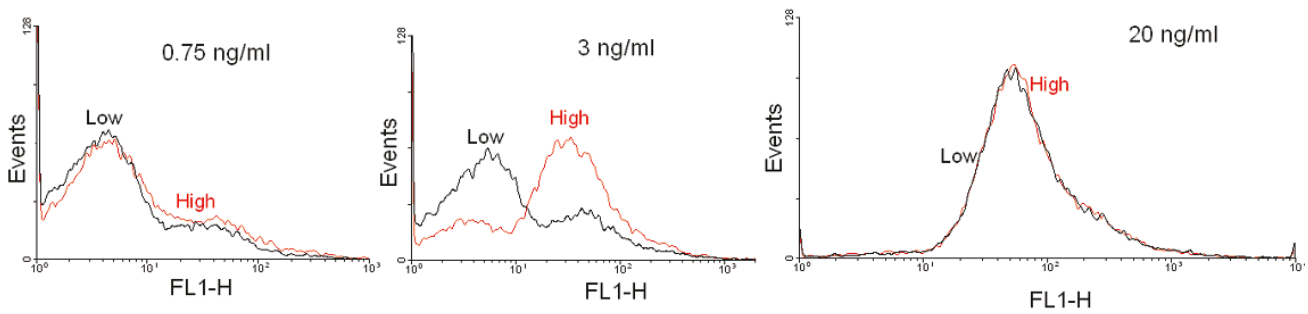

(b)

Figure 8 Hysteresis via GFP distributions. The distributions in the stationary phase with two different histories (see text) when gfp is fused with (a) $\mathrm{mprA}$ and (b) sigE promoter. The specific inducer concentrations are mentioned with each plot.

in mycobacteria [46-49]. A double positive feedback loop arises due to the activation of transcription initiation of sigE by MprA-P and the activation of the transcription of the mprAB operon by the SigE-RNAP complex. Posttranslational regulation of SigE is mediated by RseA, an anti-sigma factor.

Barik et al. [49] have identified a novel positive feedback involving SigE and RseA which becomes functional under surface stress. More experiments need to be carried out to obtain insight on the intricate control mechanisms at work when mycobacteria are subjected to stresses like nutrient deprivation. This will lead to a better understanding of the major contributory factors towards the generation of phenotypic heterogeneity in mycobacterial populations subjected to stress.

\section{Conclusions}

In the present study, we have characterised quantatively the single cell promoter activity of three key genes in the stringent response pathway of the mycobacterial population M. smegmatis. Under nutrient depletion, a "responsive switching" occurs from the $\mathrm{L}$ to the $\mathrm{H}$ subpopulation with low and high expression levels respectively. A comprehensive analysis of the flow cytometry data demonstrates the coexistence of two subpopulations with overlapping protein distributions. We have further established that the GFP distribution at any time point is a linear superposition of a Gaussian and a lognormal distribution. The coefficients in the linear combination depend on time whereas the component distributions are time-invariant. The Gaussian and lognormal distributions describe the distribution of protein levels in the $\mathrm{L}$ and $\mathrm{H}$ subpopulations respectively. The two distributions overlap in a range of GFP fluorescence intensity values. We also find that the experimental data for the $\mathrm{H}$ subpopulation can be fitted very well by the gamma distribution though the lognormal distribution gives a slightly better fit. In the case of skewed positive data sets, the two distributions are often interchangeable [50]. An analytical framework similar to that in Ref. [43] is, however, yet to be developed for the mycobacterial stringent response pathway studied in the paper. The major components in the pathway are the two-component system mprAB and multiple positive feedback loops. The two-component system is known to promote robust input-output relations [51] and persistence of gene expression states [52] which may partly explain the good fitting of the experimental data by well-known distributions. Further quantitative measurements combined with appropriate stochastic modeling are needed to characterise the experimentally observed subpopulations more uniquely. We used the binning algorithm developed in [42] to partition the experimental cell population into the $\mathrm{L}$ and $\mathrm{H}$ subpopulations. This enabled us to compute quantities like the mean protein level in the L subpopulation, the fraction of cells in the 
$\mathrm{H}$ subpopulation and the CV of GFP levels in the L subpopulation as a function of time. The picture that emerges from the analysis of experimental data is that of bistability, i.e., the coexistence of two distinct subpopulations and stochastic transitions between the subpopulations resulting in the time evolution of the fraction of cells in the $\mathrm{H}$ subpopulation. As pointed out in the earlier study [19], the rate of transition to the H subpopulation and the $\mathrm{CV}$ of the $\mathrm{L}$ subpopulation levels attain their maximum values around the same time point (Figure 5(c)) indicating the role of gene expression noise in bringing about the transition from the $\mathrm{L}$ to the $\mathrm{H}$ subpopulation. We have not attempted to develop theoretical models describing the time evolution of the relative weights, $\omega_{i}^{\prime}$ 's $(i=1,2)$, of the two subpopulations (Eq. (9)). A simple model of two interacting and evolving subpopulations with linear first order kinetics [12], cannot explain the sigmoidal nature of the time evolution. A model with nonlinear growth kinetics has been proposed in [42] but lacking definitive knowledge on the origin of nonlinearity in the growth of mycobacterial subpopulations we defer the task of model building to a future publication.

\section{Methods}

\section{Strains}

M. smegmatis $\mathrm{mc}^{2} 155$ was grown routinely in Middle Brook (MB) 7H9 broth (BD Biosciences) medium supplemented with $2 \%$ glucose and $0.05 \%$ Tween 80 .

\section{Construction of plasmids for fluorescence measurements} The mprAB promoter was amplified from the genomic DNA of $M$. smegmatis using the sense and antisense primers, 5'-AAGGTACCGCGCAACACCACAAAAAGCG3' and 5'-TAGGATCCAGTTTTGACTCACTATCTGAG-3' respectively and cloned into the promoter-less replicative $g f p$ vector pFPV27 between the KpnI and BamHI sites (in bold). The sigE and rel promoters fused to $g f p$ have been described earlier [19,31]. The resulting plasmids were electroporated into M. smegmatis $\mathrm{mc}^{2} 155$ for further study. For the study of hysteresis, expression of $p p k 1$ under a tetracycline-inducible promoter in an $M$. smegmatis strain inactivated in the ppk1 gene (PPK-KO), has been described earlier [19].

\section{FACS analysis}

M. smegmatis cells expressing different promoters fused to GFP were grown in medium supplemented with kanamycin $(25 \mu \mathrm{g} / \mathrm{ml})$ and analysed at different points of time on a FACS Caliber (BD Biosciences) flow cytometer as described earlier [19]. Briefly, cells were washed, resuspended in PBS and fluorescence intensity of 20,000 events was measured. The data was analyzed using Cell Quest Pro (BD Biosciences) and WINMIDI software. The flow cytometry data is represented in histogram plots where the $\mathrm{x}$-axis is a measure of fluorescence intensity and the $y$-axis represents the number of events.

\section{Measurement of growth rate}

$M$. smegmatis expressing promoter-gfp fusion constructs were grown in Middle Brook (MB) 7H9 broth supplemented with glucose and Tween 80, and kanamycin $(25 \mu \mathrm{g} / \mathrm{ml})$. Growth at different time points was measured by recording absorbance values at $600 \mathrm{~nm}$ (a value of $1 \mathrm{OD}_{600}$ is equal to $10^{8}$ cells or $200 \mu \mathrm{g}$ dry weight of cells). A growth curve was generated by plotting absorbance values against time (inset of Figure 2). The specific growth rate $\mu$ (Eq. (2)) at different time points is determined by taking derivatives of the growth curve at the different time points (Figure 2).

\section{Additional material}

Additional file 1: Supplementary Information. Contains description of mathematical model including Figures S1-S6.
Acknowledgements

IB, JB and MK thank M. Thattai for some useful discussions. This work was supported in part by a grant from the Department of Biotechnology, Government of India to MK. SG is supported by CSIR, India, under Grant No. 09/015(0361)/2009-EMR-I.

\section{Author details}

${ }^{1}$ Department of Physics, Bose Institute, Kolkata, India. ${ }^{2}$ Department of Chemistry, Bose Institute, Kolkata, India. ${ }^{3}$ Centre for Applied Mathematics and Computational Science, Saha Institute of Nuclear Physics, Kolkata, India.

\section{Authors' contributions}

$\mathrm{IB}, \mathrm{JB}$ and MK conceptualised, supervised and coordinated the study. KS, JB and MK carried out the experiments. SG, BG and IB developed the theoretical model, performed the data analysis and interpreted the data. IB drafted the manuscript. All authors read and approved of the final version.

Received: 7 July 2010 Accepted: 27 January 2011

Published: 27 January 2011

\section{References}

1. Veening JW, Smits WK, Kuipers OP: Bistability, epigenetics and bethedging in bacteria. Annu Rev Microbiol 2008, 62:193-210.

2. Davidson CJ, Surette MG: Individuality in bacteria. Annu Rev Genet 2008, 42:11.1-11.6.

3. Fraser D, Kaern M: A chance at survival: gene expression noise and phenotypic diversification strategies. Mol Microbiol 2009, 71:1333-1340.

4. Locke JCW, Elowitz MB: Using movies to analyse gene circuit dynamics in single cells. Nat Rev Microbiol 2009, 7:383-392.

5. Dubnau D, Losick R: Bistability in bacteria. Mol Microbiol 2006, 61:564-572.

6. Smits WK, Kuipers OP, Veening JW: Phenotypic variation in bacteria: the role of feedback regulation. Nat Rev Microbiol 2006, 4:259-271.

7. Kussell E, Leibler S: Phenotypic diversity, population growth and information in fluctuating environments. Science 2005, 309:2075-2078.

8. Leisner M, Stingl K, Frey E, Maier B: Stochastic switching to competence. Curr Opin Microbiol 2008, 11:553-559.

9. Balaban NQ, Merrin J, Chait R, Kowalik L, Leibler S: Bacterial persistence as a phenotypic switch. Science 2008, 305:1622-1625. 
10. Gefen O, Balaban NQ: The importance of being persistent: heterogeneity of bacterial populations under antibiotic stress. FEMS Microbiol Rev 2009, 33:704-717.

11. Dhar N, McKinney JD: Microbial phenotypic heterogeneity and antibiotic tolerance. Curr Opin Microbiol 2007, 10:30-38.

12. Thattai $M$, van Oudenaarden A: Stochastic gene expression in fluctuating environments. Genetics 2004, 167:523-530.

13. Acar M, Mettetal JT, van Oudenaarden A: Stochastic switching as a survival strategy in fluctuating environments. Nat Genet 2008, 40:471-479.

14. Arkin A, Ross J, McAdams HH: Stochastic kinetic analysis of developmental pathway bifurcation in phage $\lambda$-infected $E$. coli cells. Genetics 1998, 149:1633-1648

15. Ozbudak EM, Thattai M, Lim HN, Shraiman BI, van Oudenaarden A: Multistability in the lactose utilization network of E. coli. Nature 2004, 427:737-740

16. Acar M, Becskei M, van Oudenaarden A: Enhancement of cellular memory by reducing stochastic transitions. Nature 2004, 435:228-232.

17. Süel G, Garcia-Ojalvo J, Liberman LM, Elowitz M: An excitable gene regulatory circuit induces transient cellular differentiation. Nature 2006, 440:545-550.

18. Maamar $H$, Raj A, Dubnau D: Noise in gene expression determines cell fate in B. subtilis. Science 2007, 317:526-529.

19. Sureka K, Ghosh B, Dasgupta A, Basu J, Kundu M, Bose I: Positive feedback and noise activate the stringent response regulator Rel in mycobacteria. PLOS ONE 2008, 3:e1771.

20. McCune RM, Feldman FM, Lambert HP, McDermont W: Microbial persistence. II. Characteristics of the sterile state of tubercle bacilli. J Exp Med 1966, 123:445-468.

21. Lewis K: Persister cells, dormancy and infections disease. Nat Rev Microbiol 2007, 5:48-56.

22. Young D, Stark J, Kirschner D: Systems biology of persistent infection: tuberculosis as a case study. Nat Rev Microbiol 2008, 6:520-528.

23. Bishai W: Lipid lunch for persistent pathogen. Nature 2000, 406:683-685.

24. De Voss JJ, Rutter K, Schroeder BG, Barry CE III: Iron acquisition and metabolism by mycobacteria. J Bacteriol 1999, 181:4443-4451.

25. Nyka W: Studies on the effect of starvation on mycobacteria. Infect Immun 1974, 9:843-850.

26. Smeulders MJ, Keer J, Speight RA, Williams HD: Adaptation of Mycobacterium smegmatis to stationary phase. J Bacteriol 1999, 181:270-283.

27. Braeken $K$, Moris M, Daniels R, Vanderleyden J, Michiels J: New horizons for (p)ppGpp in bacterial and plant physiology. Trends Microbiol 2006, 14:45-54.

28. Primm TP, Andersen SJ, Mizrahi V, Avarbock D, Rubin $H$, et al: The stringent response of Mycobacterium tuberculosis is required for long-term survival. J Bacteriol 2000, 182:4889-4898.

29. Dahl $J$, Kraus CN, Bosho HIM, Doan B, Foley K, et al: The role of RelMtbmediated adaptation to stationary phase in long-term persistence of Mycobacterium tuberculosis in mice. Proc Natl Acad Sci USA 2003, 100:10026-10031.

30. Ojha AK, Mukherjee TK, Chatterji D: High intracellular level of guanosine tetraphosphate in Mycobacterium smegmatis changes the morphology of the bacterium. Infect Immun 2000, 68:4084-4091.

31. Sureka K, Dey S, Datta P, Singh AK, Dasgupta A, et al: Polyphosphate kinase is involved in stress-induced mprAB-sigE-rel signaling in mycobacteria. Mol Microbiol 2007, 65:261-276.

32. Tan C, Marguet P, You L: Emergent bistability by a growth-modulating positive feedback circuit. Nat Chem Biol 2009, 5:842-848.

33. Klumpp S, Zhang Z, Hwa T: Growth rate-dependent global effects on gene expression in bacteria. Cell 2009, 139:1366-1375.

34. Glover RT, Kriakov J, Garforth SJ, Baughn AD, Jacobs WRJ: The twocomponent regulatory system senX3-regX3 regulates phosphatedependent gene expression in Mycobacterium smegmatis. J Bacteriol 2007, 189:5495-5503.

35. Rifat D, Bishai WR, Karakousis PC: Phosphate Depletion: A Novel Trigger for Mycobacterium tuberculosis Persistence. J Infect Dis 2009, 200:1126-1135.

36. López-Maury L, Marguerat S, Bähler J: Tuning gene expression to changing environments: from rapid responses to evolutionary adaptation. Nat Rev Genet 2008, 9:583-593.
37. Monod J: The growth of bacterial cultures. Annu Rev Microbiol 1949, 3:371-394.

38. Zhart TC, Deretic V: Mycobacterium tuberculosis signal transduction system required for persistent infections. Proc natl Acad Sci USA 2001 98:12706-12711.

39. Pang $X$, Phong V, Thomas FB, Saleena G, et al: Evidence for complex interactions of stress-associated regulons in an mprAB deletion mutant of Mycobacterium tuberculosis. Microbiology 2007, 153:1229-1242.

40. Kaern M, Elston TC, Blake WJ, Collins JJ: Stochasticity in gene expression: from theories to phenotypes. Nat Rev Genet 2005, 6:451-464

41. Raj A, van Oudenaarden A: Nature, Nurture, or Chance: Stochastic Gene Expression and Its Consequences. Cell 2008, 135:216-226.

42. Chang $\mathrm{HH}$, Hemberg M, Barahona M, Ingber DE, Huang S: Transcriptomewide noise controls lineage choice in mammalian progenitor cells. Nature 2008, 453:544-547.

43. Friedman N, Cai L, Xie XS: Linking Stochastic Dynamics to Population Distribution: An Analytical Framework of Gene Expression. Phys Rev Lett 2006, 97:168302(1)-168302(4).

44. Cai L, Friedman N, S XX: Stochastic protein expression in individual cells at the single molecule level. Nature 2006, 440:358-362.

45. Taniguchi Y, Choi PJ, Li GW, Chen H, Babu M, et al: Quantifying E. coli Proteome and Transcriptome with Single-Molecule Sensitivity in Single Cells. Science 2010, 329(5991):533-538.

46. Donà V, Rodrigue $S$, Dainese $E$, Palù $G$, Gaudreau L, Manganelli R, Provvedi R: Evidence of Complex Transcriptional, Translational, and Posttranslational Regulation of the Extracytoplasmic Function Sigma Factor $\sigma^{E}$ in Mycobacterium tuberculosis. J Bacteriol 2008, 190:5963-5971.

47. Rodrigue S, Provvedi R, Jacques PE, Gaudreau L, Manganelli R: The sigma factors of Mycobacterium tuberculosis. FEMS Microbiol Rev 2006, 30:926-941.

48. He H, Hovey R, Kane J, Singh V, Zahrt TC: MprAB is a stress-responsive two-component system that directly regulates expression of sigma factors SigB and SigE in Mycobacterium tuberculosis. J Bacteriol 2006, 188:2134-2143

49. Barik S, Sureka K, Mukherjee P, Basu J, Kundu M: RseA, the SigE specific anti-sigma factor of Mycobacterium tuberculosis, is inactivated by phosphorylation-dependent ClpC1P2 proteolysis. Mol Microbiol 2009, 75:592-606.

50. Wiens BL: When log-normal and gamma models give different results: a case study. The American Statistician 1999, 53(2):89-93.

51. Shinar G, R M, Martinez MR, U A: Input-output robustness in simple bacterial signaling systems. PNAS 2007, 104:19931-35.

52. Kato A, Mitrophanov AY, Groisman EA: A connector of two-component regulatory systems promotes signal amplification and persistence of expression. PNAS 2007, 104:12063-68.

doi:10.1186/1752-0509-5-18

Cite this article as: Ghosh et al:: Phenotypic heterogeneity in mycobacterial stringent response. BMC Systems Biology 2011 5:18.

\section{Submit your next manuscript to BioMed Central and take full advantage of:}

- Convenient online submission

- Thorough peer review

- No space constraints or color figure charges

- Immediate publication on acceptance

- Inclusion in PubMed, CAS, Scopus and Google Scholar

- Research which is freely available for redistribution

Submit your manuscript at www.biomedcentral.com/submit
C Biomed Central 